\title{
Dépistage du cancer colorectal: ce que nous gagnons
}

\section{Urs Marbet}

Prof. Dr méd., senior consultant Hôpital cantonal d'Uri, membre FMH

\begin{abstract}
Le cancer du côlon fait partie des cancers potentiellement mortels les plus fréquents en Suisse. Les chimiothérapies, la radiothérapie, les opérations, les anus artificiels entraînent une détérioration massive de la qualité de vie et occasionnent des coûts importants. Le dépistage du cancer colorectal prévient de nombreuses complications, et un diagnostic précoce augmente la durée et la qualité de vie, mais à condition que la coloscopie soit d'excellente qualité.
\end{abstract}

\section{Résumé}

Lorsqu'il n'est détecté qu'à la suite de douleurs, le cancer colorectal est souvent déjà à un stade avancé et les chances de survie sont réduites. Le traitement - opérations, chimiothérapies et radiothérapie - est alors particulièrement lourd pour les patients et, en outre, très onéreux.

Les coloscopies effectuées dans le cadre d'examens de dépistage permettent de diagnostiquer le cancer du côlon le plus souvent à un stade précoce et d'améliorer les chances de guérison. II n'est pas rare de pouvoir procéder à une ablation endoscopique immédiate des tumeurs. Par ailleurs, I'ablation des lésions précancéreuses empêche souvent le développement du cancer et rend tout traitement supplémentaire superflu. La condition à un dépistage efficace et économique est la haute qualité de la coloscopie: un taux de dépistage élevé des lésions précancéreuses implique d'excellents instruments ainsi qu'une préparation minutieuse de l'intestin avec une phase de retrait suffisamment lente. Ces standards de qualité doivent être défendus face aux efforts d'économies. Des adénomes oubliés et une augmentation des cancers colorectaux reviendraient en effet cher aux patients, mais aussi à toute la société.

\section{Quel est l'intérêt pour le patient de la coloscopie?}

Monsieur ZA, âgé de 69 ans, qui ne présente aucun symptôme ni antécédents familiaux, a récemment subit une coloscopie. Cet examen a montré un adénome de plusieurs centimètres, une lésion précancéreuse située à proximité de l'anus, ainsi que plusieurs adénomes sur le gros intestin qui ont été enlevés lors de la coloscopie. Si Monsieur ZA avait attendu d'avoir des douleurs pour consulter, comme cela était le cas il y a quelques années faute d'alternative, il aurait été nécessaire de l'opérer, de lui implanter un anus artificiel, et le patient serait décédé plus tard, selon toute probabilité, de son cancer colorectal.

\section{Le dépistage du cancer colorectal améliore les chances de survie pour une maladie fréquente}

Chaque année en Suisse, près de 4100 personnes développent un cancer du côlon et 1600 d'entre elles en meurent [1]. Si l'on considère les années de vie perdues à cause d'un cancer, le cancer colorectal occupe la troisième place [1]. Comme le taux de maladies augmente avec l'âge, le cancer du côlon et sa prévention ont pris une importance croissante dans notre société vieillissante.

Depuis une bonne quinzaine d'années, le canton d'Uri enregistre toutes les personnes chez lesquelles un cancer colorectal a été diagnostiqué en distinguant si ce diagnostic résulte d'un dépistage du cancer colorectal ou de douleurs ayant entraîné la coloscopie. Les données permettent de dresser un tableau très clair: chez les personnes qui ont fait une coloscopie suite à des douleurs, plus de $80 \%$ ont malheureusement déjà dépassé le stade précoce facile à traiter; la plupart d'entre elles présentent déjà des infiltrations dans les ganglions lymphatiques et souvent des métastases dans d'autres organes [2,3]. Chez les personnes ne présentant aucune douleur en revanche, le cancer colorectal est dépisté dans $70 \%$ des cas au stade I, la forme la plus précoce avec de fortes chances de guérison. Tandis que $40 \%$ des patients n'ayant pas subi de dépistage décèdent plus 
tard du cancer, le taux de mortalité n'atteint pas 10\% chez les patients diagnostiqués dans le cadre d'un examen de dépistage.

\section{Le dépistage du cancer colorectal prévient la maladie et économise des traitements}

Le traitement du cancer colorectal est lourd et onéreux. Grâce à son dépistage, il se fait de plus en plus rare: la coloscopie permet d'enlever de nombreux adénomes, soit des lésions précancéreuses, et de prévenir souvent ce type de cancer. Nos données montrent qu'une coloscopie effectuée à titre préventif a permis d'éviter 8 à 10 carcinomes chez 1000 personnes de plus de 50 ans en l'espace de dix ans [3]. Aux Etats-Unis où le dépistage du cancer colorectal est très répandu depuis des années, le nombre des nouveaux cas de cancer du côlon chez les plus de 55 ans a diminué de moitié [4]. En Suisse où le 2013, cette tendance est également perceptible du moins pour le cancer colorectal distal [5].

Aujourd'hui, il n'est pas rare que le cancer du côlon au stade I, le stade rencontré dans la plupart des cas de dépistage, puisse être simplement enlevé par voie endoscopique, ce qui était impensable auparavant. Une radiothérapie ou une chimiothérapie est alors évitée. Même si aujourd'hui les patients atteints d'un cancer colorectal vivent plus longtemps qu'il y a encore quelques années, ils doivent subir des chimiothérapies, des radiothérapies et des interventions chirurgicales pour enlever des métastases, fréquemment au niveau du foie et des poumons. Cela se répercute sur les coûts de santé: le traitement d'un cancer colorectal dépisté seulement suite à l'apparition de douleurs coûte aujourd'hui en moyenne CHF 100000 , tendance à la hausse en raison de thérapeutiques toujours plus performantes et onéreuses. Il ne s'agit donc plus aujourd'hui de savoir si le dépistage du cancer colorectal peut économiser des coûts, mais combien il permet d'économiser. Ce potentiel dépend également de l'acceptation et de l'adhésion de la population au programme de dépistage. Comme l'examen des selles par exemple est mieux accepté que la coloscopie, des programmes de prévention, qui proposeraient aux personnes présentant un faible risque de cancer un examen des selles moderne et efficace, permettraient d'économiser des coûts.

\section{Préalable à une prévention efficace: une haute qualité}

La qualité élevée de la coloscopie est le principal préalable à une prévention efficace pour prévenir l'apparidépistage du cancer colorectal est pris en charge depuis

tion de tumeurs et améliorer les chances de survie. La qualité d'une coloscopie inclut la parfaite préparation de l'intestin, l'examen compétent par des endoscopeurs bien formés et dotés d'excellents instruments de haute technologie, l'ablation simultanée et intégrale des lésions précancéreuses, un suivi optimal des patients détectés positifs ou aux antécédents familiaux ainsi qu'un taux de participation élevé aux coloscopies et aux programmes d'examen des selles. Il a été largement démontré qu'une bonne qualité va de pair avec un taux de dépistage élevé d'adénomes. Dans l'intestin proximal notamment, des tumeurs et leurs lésions précancéreuses insidieuses (adénomes dentelés) peuvent facilement passer inaperçues même lors d'une coloscopie. Face à l'amélioration du taux de survie grâce aux coloscopies de qualité, à la réduction du stress pour les patients et à l'économie de coûts, cette qualité devrait être valorisée. En plus des instruments excellents et indispensables, coûteux à l'achat et à l'entretien, cette qualité implique un examen minutieux et approfondi afin de dépister les adénomes cachés et quasi invisibles. Des études à grande échelle menées à travers le monde ont démontré que le cancer colorectal est d'autant plus rare après une coloscopie que le nombre d'adénomes enlevés lors de l'examen était important. Plusieurs études ont établi par ailleurs que la minutie nécessaire est en corrélation directe avec le temps consacré à la coloscopie. La durée de l'examen est donc directement liée à son efficacité, et donc au bénéfice qu'il apporte aux patients. C'est pourquoi une durée minimale a été définie et la mesure du temps de retrait de l'instrument a été établie pour chaque examen. Il convient de respecter ces standards et de les défendre face aux mesures d'économies; limiter de manière irréaliste la durée d'une coloscopie implique automatiquement des examens trop rapides voire bâclés, ce qui fait perdre au dépistage du cancer colorectal toute son utilité: un cancer colorectal résultant d'adénomes ou de tumeurs non détectés représente un coût important, non seulement pour les patients et leur entourage, mais aussi pour la société.

\section{Références}

1 Office fédéral de la statistique (Hrsg.). Le cancer en Suisse, rapport 2015. Etat des lieux et évolutions. Neuchâtel, 2016.

2 Marbet UA, Bauerfeind P, Brunner J, Dorta G, Valloton JJ, Delco F: Colonoscopy is the preferred colorectal cancer screening method in a population-based programm. Endoscopy 2008;40:650-5.

3 Manser CN, Bachmann LM, Brunner J, Hunold F, Bauerfeind P, Marbet UA: Colonoscopy screening markedly reduces the occurrence of colon carcinomas and carcinoma related death: a closed cohort study. Gastrointest Endosc 2012;76:110-7.
4 Siegel RL, Fedewa SA, Anderson WF, Miller KD, Ma J, Rosenberg PS, Jemal A: Colorectal cancer incidence patterns in the United States, 1974-2013. J Natl Cancer Inst. 2017;109:djw322 JNCI 2017

5 Lorez M, Marbet U, Arndt V: subsite-specific colorectal cancer trends in Switzerland (1989-2012). Bulletin suisse du cancer 2016;1:67-4. 